\title{
Monitoring Compliance and Acceptability of Intermittent Preventive Treatment of Malaria Using Sulfadoxine Pyrimethamine after Ten Years of Implementation in Tanzania
}

\author{
Mdetele B. Ayubu and Winifrida B. Kidima \\ College of Natural and Applied Sciences, University of Dar es Salaam, P.O. Box 35064, Dar es Salaam, Tanzania \\ Correspondence should be addressed to Winifrida B. Kidima; kidimaw@udsm.ac.tz
}

Received 17 September 2016; Revised 6 February 2017; Accepted 19 March 2017; Published 30 March 2017

Academic Editor: Kwadwo Koram

Copyright (C) 2017 Mdetele B. Ayubu and Winifrida B. Kidima. This is an open access article distributed under the Creative Commons Attribution License, which permits unrestricted use, distribution, and reproduction in any medium, provided the original work is properly cited.

\begin{abstract}
Intermittent preventive treatment using SP (IPTp-SP) is still a superior interventional approach to control malaria during pregnancy. However its rate of use has gone down tremendously in malaria endemic areas. This study forms part of a larger study aimed at monitoring the compliance of IPTp-SP policy in malaria endemic areas of Tanzania. Two cross-sectional studies were conducted in Dar es Salaam and Njombe Regions of Tanzania. Overall, 540 pregnant women and 21 healthcare workers were interviewed using structured questionnaires. This study revealed that $63 \%$ of women were not willing to take SP during pregnancy while $91 \%$ would only take it if they tested positive for malaria during antennal visits. $63 \%$ of the interviewed women did not know the recommended dose of SP required during pregnancy, despite the fact that $82 \%$ of the women were aware of the adverse effect of malaria during pregnancy. It was found out that $54 \%$ of pregnant women (30-40 weeks) took single dose, $34 \%$ took two doses, and $16 \%$ did not take SP at the time of interview. It was also found that SP was not administered under direct observed therapy in $86 \%$ of women. There was no significant relationship between number of doses received by pregnant women and antenatal clinic (ANC) start date $\left(r^{2}=0.0033,95 \%\right.$ CI $(-0.016$ to 0.034$\left.)\right)$. However positive correlation between drug uptake and drug availability was revealed $(p=0.0001)$. Knowledge on adverse effects of placental malaria among pregnant women was significantly associated with drug uptake (OR 11.81, 95\% CI (5.755-24.23), $p=0.0001)$. We conclude that unavailability of drugs in ANC is the major reason hindering the implementation of IPTp-SP.
\end{abstract}

\section{Introduction}

Intermittent preventive treatment using Sulphadoxine Pyrimethamine (IPTp-SP) has proven to be effective in reducing the burden of malaria during pregnancy [1-5]. SP is still a prophylactic drug of choice against malaria during pregnancy. Although 2 doses of SP have been the standard for IPTp-SP, 3 doses might be more effective in reducing burden of poor pregnancy outcome due to malaria [6]. The World Health Organization recommended that women in malaria endemic areas should take a monthly dose from second trimesters till term instead of the initially recommended 2 doses [7]. Tanzania adopted IPTp-SP program since year 2000 [8] with total coverage of about 32\% [9]. According to Demographic Health Surveillance survey IPTp-SP use has been increasing with percentages of pregnant women using more than three doses increasing from 3\% in 2004 to $8 \%$ in 2015 [10]. Despite the evidence of the effectiveness of IPTp strategy using SP in reducing the adverse effects of malaria during pregnancy, IPTp-SP compliance and therefore the uptake and coverage have been reported to be low in Tanzania [11-13]. Low uptake of SP may increase rate of placental parasitaemia and hence rates of miscarriage, stillbirth, and delivering of low birth babies. WHO recommend regular monitoring of IPTp-SP among women in malaria endemic areas. Therefore there is a need to constantly monitor factors that are associated with low uptake of SP among the women in endemic areas.

Apart from IPTp-SP, insecticide treated nets (ITN) have been utilized on large scale in Tanzania and in selected places 
indoor residual spraying is being utilized to reduce humanmosquito contact. The use of ITN has accounted for about $50 \%$ reduction of malaria cases $[14,15]$. However residual malaria which is the result of mosquito behavioral change [16, 17] together with increasing mosquitoes insecticide resistance has put pregnant women at high risk to acquire malaria despite the use of ITN. Following that, the use of IPTp-SP remains critical in minimizing effect of placental malaria in endemic areas.

In this study we aimed at investigating knowledge, acceptability of IPTp-SP, and factors that affect compliance of IPTpSP in Tanzanian after more than ten years of adaptation. Specifically we assessed the proportion of women who were aware of the adverse effect of placental malaria, assessed availability of SP in public antenatal clinics, and outlined factors that are associated with low uptake of IPTp-SP by pregnant women. We also assessed the willingness of pregnant women in testing for malaria during regular ANC visits. Information from this study will help to improve strategies to increase access of SP to pregnant women.

\section{Methodology}

2.1. Study Area and Sampling Design. This study involved two cross-sectional surveys carried out in February-April 2015 and February-June 2016 in four districts: three (Ilala, Temeke, and Kinondoni) from Dar es Salaam urban, eastern coast of Tanzania, and one (Njombe District) from Iringa. Dar es Salaam $\left(6.7924^{\circ} \mathrm{S}, 39.2083^{\circ} \mathrm{E}\right)$ is bounded by the Indian Ocean on the east and by the Coast Region on the other three sides. The area experiences tropical climatic conditions, characterized by hot and humid weather throughout much of the year. Annual rainfall is approximately $1,100 \mathrm{~mm}$. Njombe is located in Iringa region, in the southern part of Tanzania. It is centered around latitude of $7.77^{\circ} \mathrm{S}$ and longitude of $35.69^{\circ} \mathrm{E}$, 1,581 meters elevation above sea level. Both Dar es Salaam and Iringa are malaria hyperendemic areas. We selected thirteen different antenatal clinics purposefully based on ANC availability.

A researcher was given an opportunity to describe the study to pregnant women attending ANC. A questionnaire with closed and open ended questions was administered to $50 \%$ of randomly selected consenting pregnant women. Information included social and demographic information, availability of SP at the clinic, ANC start date, use of ITN, gravidity, education level, malaria positivity during pregnancy, awareness of IPTp-SP program, acceptability/willingness to take SP as an malaria interventional program during pregnancy, the mode in which SP was administered (utilization of DOT), number of doses of SP received, availability of SP at the clinic, side effect observed, knowledge on adverse effect of malaria during pregnancy, recommended dosage of SP, preference to test for malaria at ANC, and participants view on improvement of IPTp-SP.

A health provider questionnaire was also administered to enquire about availability of SP at the clinic and reasons for lower uptake of IPTp-SP among pregnant women, provision of information about the IPTp-SP program, and other factors affecting uptake of SP compliance on IPTp-SP.
Research clearance for the project was obtained from the Commission for Science and Technology Tanzania (NDC/P.20/2/VOLIII/14) through the University of Dar es Salaam Vice Chancellor office. Permission to conduct this study in three districts in Dar es Salaam and Njombe was obtained from the Regional Administrative Secretary (RAS), District Medical Officer in charge of the respective facilities. Written consent from the study participant was obtained from 540 pregnant women and 21 healthcare workers.

2.2. Sample Size Calculation. The sample size calculation was based on the formula by Pfeiffer $(2002)=Z^{2} P(1-P) / d^{2}$, where $P$ is the rate of optimal uptake of IPTp-SP in Tanzania which is estimated to be $43 \%$ [11], $Z$ stands for a standard normal variate at 5 (1.96), and the absolute error or precision $d$ is 0.05 . The minimum sample size obtained was 376 . This was distributed within the two study sites. All health workers who consented were interviewed in this study.

2.3. Data Analysis. Data analysis was performed using Graph Prism version 5. Fisher exact test was used to test for association between knowledge on impact of placental malaria and drug uptake.

Linear regression was used to analyze relationship between number of doses received and ANC start date. The association between drug uptake and drug availability of SP at ANC was analyzed using Spearman's rank correlation test.

\section{Results}

3.1. Characteristics of Study Subjects. Five hundred and forty pregnant women and 21 health workers were successfully interviewed in Dar es Salaam and Njombe. The mean age range for pregnant women interviewed was 27 (15-42) years. The majority (39\%) of women were multigravidae (Table 1). $22 \%$ of women were primigravidae. Forty-seven percent of pregnant women had been to secondary schools. Only $14 \%$ of pregnant women reported having tested positive for malaria during the time of pregnancy. All women interviewed reported to have been using insecticide treated nets.

3.2. Knowledge of the Adverse Effect of Placental Malaria on Pregnancy Outcomes and the Recommended Dose of SP in IPTp. Most of the interviewed women were aware of IPTpSP as an intervention to control malaria during pregnancy (Figure 1). However, about $49 \%$ of women who were aware of IPTP- SP did not know the recommended dosage required during pregnancy to control malaria (Figure 1). In addition to the knowledge on SP dosage, a large proportion (451/540) of interviewed women did not know the effect of malaria during pregnancy (Figure 1). Effect of malaria during pregnancy outlined by $89 / 540$ women included preterm delivery $(69 \%$, $n=63)$, maternal and neonatal deaths $(13 \%, n=15)$, and congenital malaria $(17 \%, n=11)$. Using Contingency Tables it was found that knowledge on adverse effects of placental malaria among pregnant women was associated with drug uptake (odds ratio (OR) $=11.81,95 \%$ CI (5.755-24.23), $p=$ 0.0001 , Fisher exact test). 
TABLE 1: Characteristics of study subjects.

\begin{tabular}{lc}
\hline Mean age in years (range) & $27(15-42)$ \\
Gravid & Proportions\% $(n)$ \\
Primigravidae & $29(157)$ \\
Secundigravidae & $32(175)$ \\
Multigravidae & $39(208)$ \\
Weeks of pregnancy & \\
First trimester & $16(85)$ \\
Second trimester & $59(317)$ \\
Third trimester & $25(138)$ \\
Level of education & \\
No school & $12(64)$ \\
Primary education & $24(130)$ \\
Secondary education & $47(251)$ \\
Tertiary education & $16(88)$ \\
No response & $1(7)$ \\
Malaria positive during pregnancy \\
(self-reported cases)
\end{tabular}

3.3. IPTp-SP Acceptability and Compliance. Contrary to what we had predicted, it was found that a large proportion $(63 \%$, $n=340$ ) of women were not willing to take SP during pregnancy, $9 \%(n=31)$ citing fear of side effects and $91 \%$ $(n=309)$ with no clear reason(s). In addition it was found that about $91 \%(n=491)$ of women preferred to test for malaria before agreeing to take SP. Only third-trimester pregnant women (weeks 28-36) were interviewed on SP dosage received at the time of interview. In view of this only 138 responses were analyzed. About $54 \%(n=74)$ of women took single dose, $34 \%(n=50)$ took two doses, and $16 \%$ $(n=22)$ did not take SP at the 3rd trimester. Ninety-two percent $(n=68)$ of pregnant women who received a single dose declared that they were not given SP on follow-up visits. No significant relationship was found between ANC start date and dosage received (Pearson's $r^{2}=0.003261,95 \%$ CI $(-0.01639$ to 0.03376$))$. However, there was a positive correlation between drug uptake and drug availability of SP at ANC (Spearman $r=0.67, p=0.0001,95 \%$ CI $(0.54$ to $0.77)$ ). We also asked whether the SP were administered by directly observed therapy (DOT) as recommended by WHO. Overall we found DOT was not observed in 86\% (314/364) of all pregnant women who were given SP.

3.4. Health Workers Responses. A total of 21 healthcare workers from different antenatal clinics were interviewed on factors affecting uptake of SP by pregnant women. 100\%

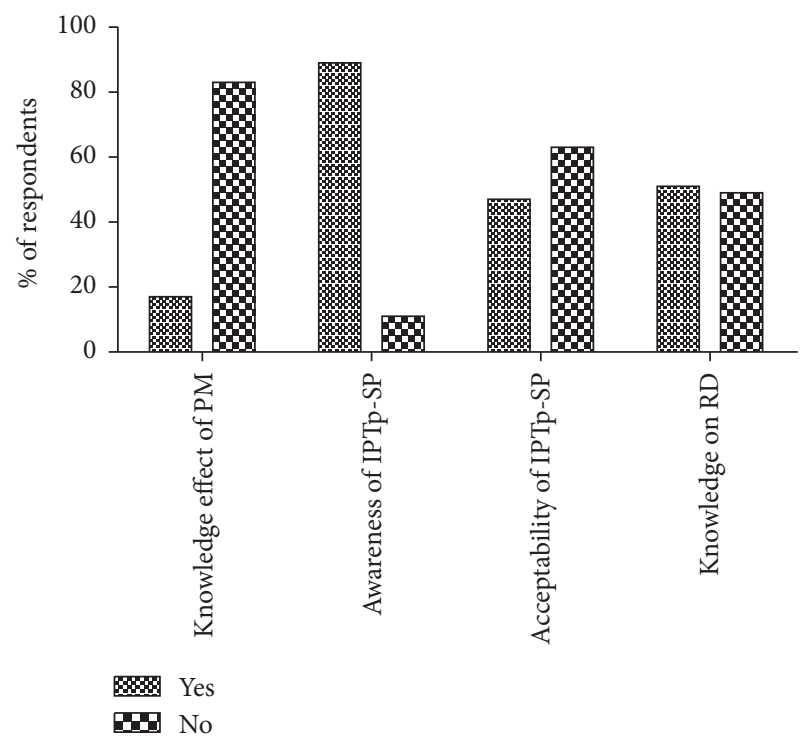

FIGURE 1: Percentage respondents (pregnant women) on knowledge of placental malaria, awareness, acceptability, and recommended dose of IPTp-SP among pregnant women in Dar es Salaam and Njombe. RD: recommended dose; PM: placental malaria $(n=540)$.

confirmed provision of information about IPTp-SP to pregnant women at the clinic. $95 \%(n=20)$ of health workers declared that SP is not always available at the clinics. We found SP was not available in $77 \%$ of antenatal clinics at the time of interview. Other reasons for low uptake of SP outlined included late attendance to antenatal clinics by some of pregnant women.

\section{Discussion}

In the present study we found that, despite high level of awareness and knowledge about IPTp-SP, the acceptability and uptake of SP among pregnant women are still low. Additionally this study establishes a link between low supply of SP in ANC, knowledge about impact of placental malaria by pregnant women, and uptake of SP. These results support the observations by Marchant et al., 2008, Mpogoro et al., 2014, and Mubyazi and Bloch, 2014, who observed SP unavailability and low uptake of SP among women in Tanzania $[13,18,19]$. Low uptake of SP may increase risk of malaria parasitaemia and therefore placental malaria prevalence among pregnant women in endemic areas.

Studies have shown that uptake of IPTp-SP has been increasing with percentages of pregnant women taking more than 3 doses rising from $3 \%$ in 2000 to $8 \%$ in 2015 [10]. Majority of the third-trimester women in our study received a single dose of SP at 28 to 40 weeks of pregnancy. Mubyazi and colleague, 2014, observed that delivery and uptake of IPTpSP were affected by late ANC attendance [13]. Surprisingly, we did not find a relationship between ANC start date and dosage of IPTp-SP received by pregnant women. However an association between availability of SP in clinics and uptake was observed in this study. Studies have shown that SP is 
not always available in clinics in other malaria endemic areas such as Nigeria $[13,20]$, suggesting a major barrier to IPTpSP implementation. Unavailability of SP in clinics may result in women taking suboptimal prophylactic doses. Multiple doses of SP have been shown to improve clinical outcomes in pregnant women in malaria endemic areas. For example, two or more doses have been shown to be effective in reducing prevalence of malaria parasitaemia [4] and therefore improve pregnancy outcomes. Constant supply line of SP drugs remains critical in facilitating uptake of SP among pregnant women in malaria endemic areas and therefore a major challenge to be addressed.

Although DOT has been shown to improve compliances to IPTp-SP, its utilization at ANCs in many malaria settings has been shown to be challenging [21]. DOT was not observed in majority of women receiving SP in this study. In spite of high level of knowledge about IPTp-SP as an intervention among the women in this study, high refusal rates were observed. In Malawi limited understanding of IPTp-SP among pregnant women affected uptake [22], emphasizing the need to provide knowledge on placental malaria and IPTp-SP program to pregnant women in endemic areas.

IPTp-SP is still a superior interventional program for controlling malaria [23] among pregnant women. A randomized control trial of IPTp-SP versus IPTp-Artemisinin Lumefantrine (AL) on women in low malaria transmission areas indicated that SP was superior in reducing malaria parasitaemia and performed equally well as IPTp-AL in improving pregnancy outcomes (reviewed in [23]). Furthermore, studies by Desai et al. 2015 reported that intermittent screening and treatment in pregnancy have no value addition compared to the use of SP alone insisting superiority of IPTp-SP during pregnancy [24]. In the current study many women preferred to test for malaria before the uptake of SP suggesting more provision of information, education, and communication about the benefits of IPTp-SP uptake.

Unfortunately we were unable to evaluate from this data the effect of low dosage of SP on pregnant outcomes among the pregnant women studied. Other limitations in our study include small number of third trimester women analyzed and the randomization procedure that may have led to sampling bias. Notwithstanding its limitation this study showed higher refusal rates of IPTp-SP among studied women. Higher refusal rates against IPTp-SP signal poor delivery of knowledge on IPTp-SP among women and calls for alternative IPTp-SP-knowledge-delivery mechanisms. We recommend that SP should be available in ANCs for successful scale-up of IPTp-SP program.

\section{Conflicts of Interest}

The authors declare that there are no conflicts of interest regarding the publication of this paper.

\section{Acknowledgments}

The authors would like to thank all pregnant women for their participation in this study. They are grateful to all the health staff for their technical support. They would also like to thank the Dar es Salaam and Iringa Regional Administrative secretary and District Medical Officers of the respective facilities for giving them permission to conduct this research.

\section{References}

[1] I. I. Daud, F. O. Opinya, D. Midem et al., "Improved pregnancy outcomes in a prospective study of pregnant women enrolling in an antenatal clinic in Western Kenya," Health, vol. 6, no. 19, pp. 2651-2656, 2014.

[2] D. Mosha, J. Chilongola, R. Ndeserua, F. Mwingira, and B. Genton, "Effectiveness of intermittent preventive treatment with sulfadoxine-pyrimethamine during pregnancy on placental malaria, maternal anaemia and birthweight in areas with high and low malaria transmission intensity in Tanzania," Tropical Medicine and International Health, vol. 19, no. 9, pp. 1048-1056, 2014.

[3] S. Gies, S. O. Coulibaly, F. T. Ouattara, and U. D’Alessandro, "Individual efficacy of intermittent preventive treatment with sulfadoxine-pyrimethamine in primi- and secundigravidae in rural Burkina Faso: impact on parasitaemia, anaemia and birth weight," Tropical Medicine and International Health, vol. 14, no. 2, pp. 174-182, 2009.

[4] L. Hommerich, C. Von Oertzen, G. Bedu-Addo et al., "Decline of placental malaria in southern Ghana after the implementation of intermittent preventive treatment in pregnancy," Malaria Journal, vol. 6, article 144, 2007.

[5] V. N. Orish, O. S. Onyeabor, J. N. Boampong et al., "Prevalence of intermittent preventive treatment with sulphadoxinepyrimethamine (IPTp-Sp) use during pregnancy and other associated factors in Sekondi-Takoradi, Ghana," African Health Sciences, vol. 15, no. 4, pp. 1087-1096, 2015.

[6] E. Arinaitwe, V. Ades, A. Walakira et al., "Intermittent preventive therapy with sulfadoxine-pyrimethamine for malaria in pregnancy: a cross-sectional study from Tororo, Uganda," PLoS ONE, vol. 8, no. 9, Article ID e73073, 2013.

[7] WHO Malaria Policy Advisory Committee and Secretariat, "Malaria Policy Advisory Committee to the WHO: conclusions and recommendations of March 2013 meeting," Malaria Journal, vol. 12, article 213, 2013.

[8] J. Crawley, J. Hill, J. Yartey et al., "From evidence to action? Challenges to policy change and programme delivery for malaria in pregnancy," The Lancet Infectious Diseases, vol. 7, no. 2, pp. 145-155, 2007.

[9] PATH, "Policies and actions for improved Malaria in Pregnancy efforts in communities," Malaria in Pregnancy Stakeholders Forum Report, 2015.

[10] H. Survey and I. Survey, “Tanzania," 2015.

[11] A. Exavery, G. Mbaruku, S. Mbuyita, A. Makemba, I. P. Kinyonge, and H. Kweka, "Factors affecting uptake of optimal doses of sulphadoxine-pyrimethamine for intermittent preventive treatment of malaria in pregnancy in six districts of Tanzania," Malaria Journal, vol. 13, article 22, 9 pages, 2014.

[12] S. M. Kibusi, E. Kimunai, and C. S. Hines, "Predictors for uptake of intermittent preventive treatment of malaria in pregnancy (IPTp) in Tanzania," BMC Public Health, vol. 15, article 540, 2015. 
[13] G. M. Mubyazi and P. Bloch, "Psychosocial, behavioural and health system barriers to delivery and uptake of intermittent preventive treatment of malaria in pregnancy in Tanzania-viewpoints of service providers in Mkuranga and Mufindi districts," BMC Health Services Research, vol. 14, article 15, 2014.

[14] N. Protopopoff, A. Wright, P. A. West et al., "Correction: Combination of insecticide treated nets and indoor residual spraying in Northern Tanzania provides additional reduction in vector population density and Malaria transmission rates compared to insecticide treated nets alone: a randomised control trial," PLoS ONE, vol. 11, no. 1, Article ID e0146629, 2016.

[15] S. Bhatt, D. J. Weiss, E. Cameron et al., "The effect of malaria control on Plasmodium falciparum in Africa between 2000 and 2015," Nature, vol. 526, no. 7572, pp. 207-211, 2015.

[16] L. Durnez and M. Coosemans, "Residual transmission of malaria: an old issue for new approaches," in Anopheles Mosquitoes-New Insights into Malaria Vectors, S. Manguin, Ed., pp. 671-704, InTech, 2013.

[17] G. F. Killeen, "Characterizing, controlling and eliminating residual malaria transmission," Malaria Journal, vol. 13, no. 1, article 330, 2014.

[18] T. Marchant, R. Nathan, C. Jones et al., "Individual, facility and policy level influences on national coverage estimates for intermittent preventive treatment of malaria in pregnancy in Tanzania," Malaria Journal, vol. 7, article 260, 2008.

[19] F. J. Mpogoro, D. Matovelo, A. Dosani, S. Ngallaba, M. Mugono, and H. D. Mazigo, "Uptake of intermittent preventive treatment with sulphadoxine-pyrimethamine for malaria during pregnancy and pregnancy outcomes: a cross-sectional study in Geita district, North-Western Tanzania," Malaria Journal, vol. 13, no. 1, article 455, pp. 1-14, 2014.

[20] S. O. Akinleye, C. O. Falade, and I. O. Ajayi, "Knowledge and utilization of intermittent preventive treatment for malaria among pregnant women attending antenatal clinics in primary health care centers in rural southwest, Nigeria: a cross-sectional study," BMC Pregnancy and Childbirth, vol. 9, article 28, 2009.

[21] G. M. Mubyazi, P. Magnussen, C. Goodman et al., "Implementing intermittent preventive treatment for malaria in pregnancy: review of prospects, achievements, challenges and agenda for research," The Open Tropical Medicine Journal, vol. 1, pp. 92-100, 2008.

[22] A. Launiala and M.-L. Honkasalo, "Ethnographic study of factors influencing compliance to intermittent preventive treatment of malaria during pregnancy among Yao women in rural Malawi," Transactions of the Royal Society of Tropical Medicine and Hygiene, vol. 101, no. 10, pp. 980-989, 2007.

[23] S. Africa, Intermittent screening and treatment in pregnancy and the safety of ACTs in the first trimester, 2015.

[24] M. Desai, J. Gutman, A. L'Lanziva et al., "Intermittent screening and treatment or intermittent preventive treatment with dihydroartemisinin-piperaquine versus intermittent preventive treatment with sulfadoxine-pyrimethamine for the control of malaria during pregnancy in western Kenya: an openlabel, three-group, randomised controlled superiority trial," The Lancet, vol. 386, no. 10012, pp. 2507-2519, 2015. 


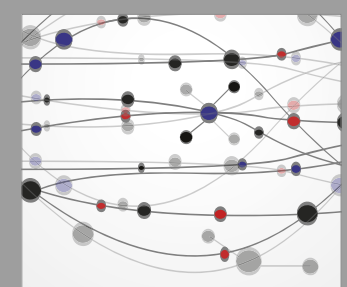

The Scientific World Journal
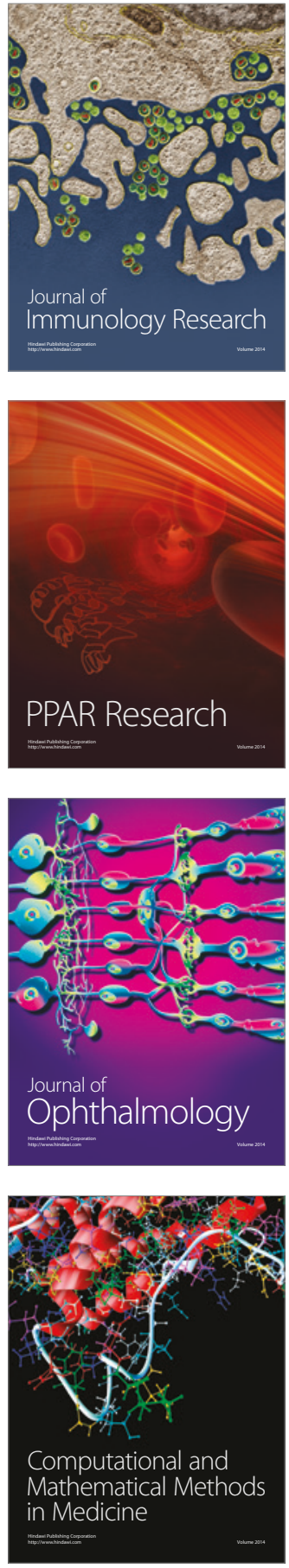

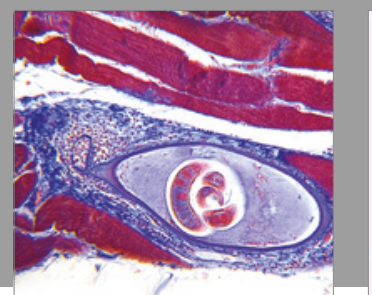

Gastroenterology Research and Practice
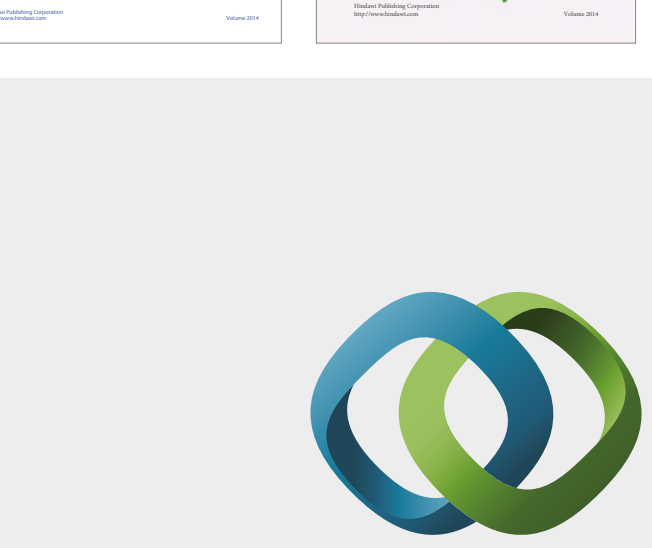

\section{Hindawi}

Submit your manuscripts at

https://www.hindawi.com
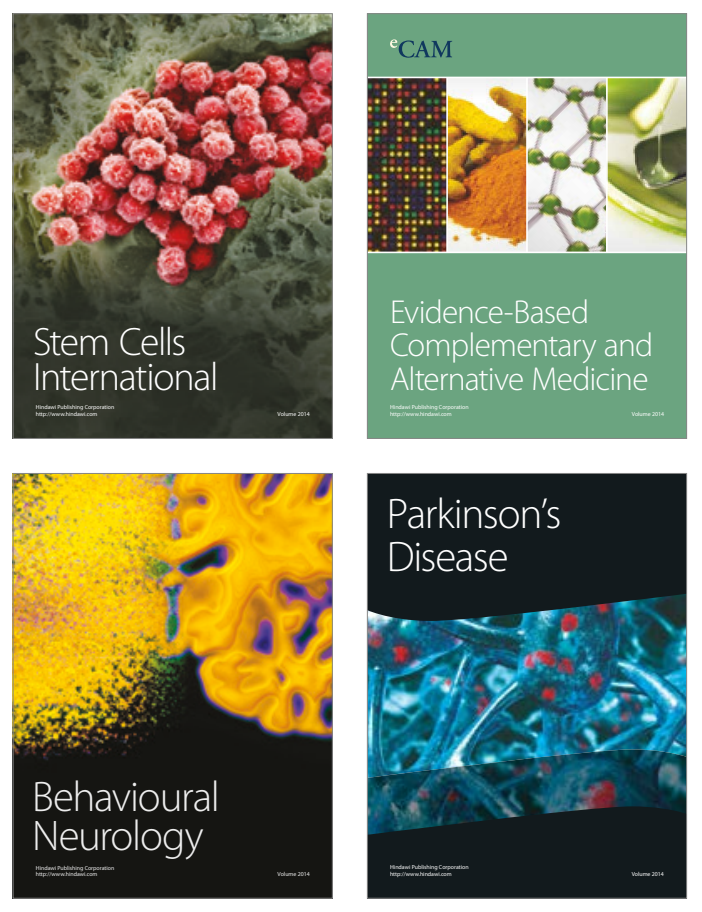
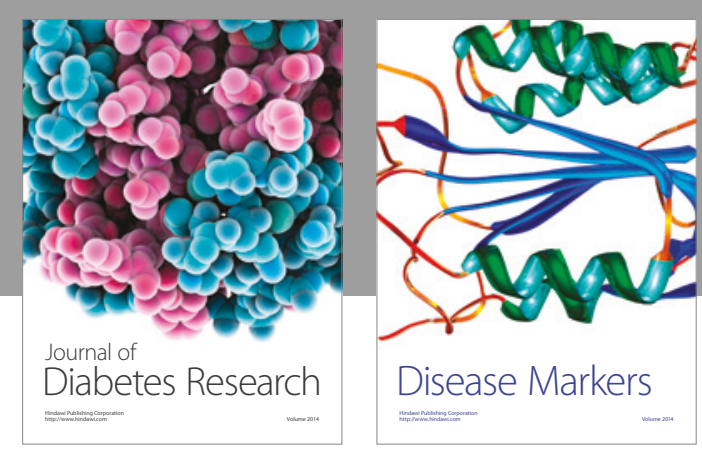

Disease Markers
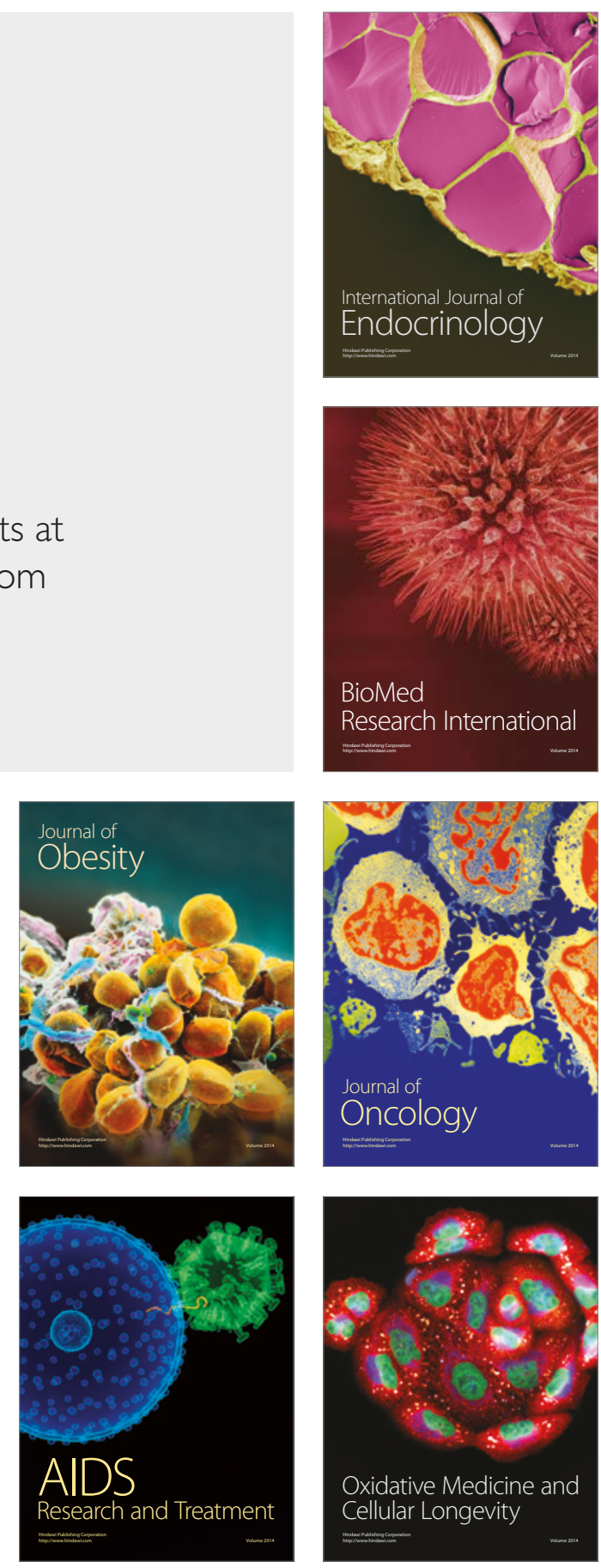